\title{
Numerical Solution of Riccati Equations by the Adomian and Asymptotic Decomposition Methods over Extended Domains
}

\author{
Jafar Biazar $^{1,2}$ and Mohsen Didgar ${ }^{2,3}$ \\ ${ }^{1}$ Department of Applied Mathematics, Faculty of Mathematical Sciences, University of Guilan, P.O. Box 41335-1914, Rasht, Iran \\ ${ }^{2}$ Department of Mathematics, Rasht Branch, Islamic Azad University, P.O. Box 41335-3516, Rasht, Iran \\ ${ }^{3}$ Department of Mathematics, Guilan Science and Research Branch, Islamic Azad University, Rasht, Iran
}

Correspondence should be addressed to Mohsen Didgar; mohsen_didgar@yahoo.com

Received 8 July 2015; Accepted 27 August 2015

Academic Editor: Timothy R. Marchant

Copyright (C) 2015 J. Biazar and M. Didgar. This is an open access article distributed under the Creative Commons Attribution License, which permits unrestricted use, distribution, and reproduction in any medium, provided the original work is properly cited.

\begin{abstract}
We combine the Adomian decomposition method (ADM) and Adomian's asymptotic decomposition method (AADM) for solving Riccati equations. We investigate the approximate global solution by matching the near-field approximation derived from the Adomian decomposition method with the far-field approximation derived from Adomian's asymptotic decomposition method for Riccati equations and in such cases when we do not find any region of overlap between the obtained approximate solutions by the two proposed methods, we connect the two approximations by the Padé approximant of the near-field approximation. We illustrate the efficiency of the technique for several specific examples of the Riccati equation for which the exact solution is known in advance.
\end{abstract}

\section{Introduction}

It is well known that the Riccati equation as

$$
u^{\prime}(x)=p(x)+q(x) u(x)+r(x) u^{2}(x)
$$

finds surprisingly many applications in physics and mathematics such as random processes, optimal control, and diffusion problems [1]. In fact, the Riccati equation naturally arises in many fields of quantum mechanics, such as in quantum chemistry [2], the Wentzel-Kramers-Brillouin approximation [3], and super symmetry theories [4]. In addition, the Riccati equation plays a prominent role in variational calculus [5], nonlinear physics [6], renormalization group equations for coupling constants in quantum field theories $[7,8]$, and thermodynamics [9]. It is well known that onedimensional static Schrödinger equation is closely related to the Riccati equation. Solitary wave solutions of a nonlinear partial differential equation can be expressed as a polynomial in two elementary functions satisfying a projective Riccati equation [10]. Beside important engineering and scientific applications that are well known, the newer applications include areas such as mathematical finance $[11,12]$.
Adomian and his coauthors have presented a systematic methodology for practical solution of linear or nonlinear and deterministic or stochastic operator equations, including algebraic equations, ordinary differential equations, partial differential equations, and integral and integrodifferential equations [13-18]. Adomian decomposition method is a powerful technique, which provides efficient algorithms for analytic approximate solutions and numerical simulations for real-world applications in the applied sciences and engineering. Using the $\mathrm{ADM}$, we calculate a series solution, but, in practice, we approximate the solution by a truncated series. The series sometimes coincides with the Taylor expansion of the exact solution in the neighborhood of the point $x=0$. Although the series can be rapidly convergent in a small region, it has a slower convergence rate in the wider region.

Several investigators have proposed a variety of approaches to solve the Riccati equation, approximately [19-24]. In order to obtain the global approximate solution of the Riccati equation, we combine the Pade approximant of the near-field approximation as derived from the ADM with the far-field approximation as derived from the AADM [25-28] to overcome the difficulty of a finite domain 
of convergence. Adomian introduced a variation of his decomposition method in [25] that can be used to obtain the asymptotic value of solutions. In this method, the recursion is the same as that in the ADM, but it uses a different canonical form of the differential equation such that it yields to a steady state solution of the equation. In fact, rather than nested integrations as in decomposition, we have nested differentiations, which will be expounded later. Haldar and Datta [29] applied the AADM to calculate integrals neither expressible in terms of elementary functions nor adequately tabulated.

This paper is arranged as follows. In the next section, we present a brief review of the ADM for nonlinear IVPs. In Section 3, we present a description of the AADM for solving the Riccati equation. In Section 4, we investigate several numerical examples. In Section 5, we present our conclusions and summarize our findings.

\section{Review of the Adomian Decomposition Method}

We review the salient features of the Adomian decomposition method in solving IVPs for first-order nonlinear ordinary differential equations as

$$
\begin{aligned}
\frac{d}{d x} u(x)+\alpha(x) u(x)+f(u(x)) & =g(x), \\
u\left(x_{0}\right) & =c_{0},
\end{aligned}
$$

where the functions $\alpha, g$, and $f$ are analytic.

We rewrite (2) in Adomian's usual operator-theoretic form

$$
L u+R u+N u=g,
$$

where $L=(d / d x)(\cdot)$ and then $L^{-1}=\int_{x_{0}}^{x}(\cdot) d x, R u=\alpha(x) u(x)$, and $N u=f(u(x))$. Next we rewrite (3) as

$$
L u=g-R u-N u,
$$

and we apply the integral operator $L^{-1}$ to both sides of (4):

$$
L^{-1} L u=L^{-1} g-L^{-1} R u-L^{-1} N u,
$$

where $L^{-1} L u=u-\Phi$ since $L \Phi=0$. In the case of a firstorder ordinary differential equation, we have $\Phi=u\left(x_{0}\right)=c_{0}$. Therefore

$$
u=\Phi+L^{-1} g-L^{-1} R u-L^{-1} N u .
$$

For the sake of simplicity, we define the $\gamma$ function as $\gamma=$ $\Phi+L^{-1} g$, and then, upon substitution, we obtain

$$
u=\gamma-L^{-1} R u-L^{-1} N u .
$$

In the $\mathrm{ADM}$, the solution $u(x)$ is represented by a series; say

$$
u(x)=\sum_{n=0}^{\infty} u_{n}(x)
$$

and the nonlinearity comprises the Adomian polynomials

$$
N u=\sum_{n=0}^{\infty} A_{n},
$$

where

$$
A_{n}=A_{n}\left(u_{0}, u_{1}, \ldots, u_{n}\right)
$$

is called an Adomian polynomial, which were first defined by Adomian [13] as

$$
A_{n}=\frac{1}{n !} \frac{d^{n}}{d \lambda^{n}}\left[N\left(\sum_{k=0}^{\infty} u_{k} \lambda^{k}\right)\right]_{\lambda=0}, \quad n \geq 0 .
$$

For convenient reference, we list the first five Adomian polynomials

$$
\begin{aligned}
A_{0}= & N\left(u_{0}\right), \\
A_{1}= & N^{\prime}\left(u_{0}\right) u_{1}, \\
A_{2}= & N^{\prime}\left(u_{0}\right) u_{2}+N^{\prime \prime}\left(u_{0}\right) \frac{u_{1}^{2}}{2 !}, \\
A_{3}= & N^{\prime}\left(u_{0}\right) u_{3}+N^{\prime \prime}\left(u_{0}\right) u_{1} u_{2}+N^{\prime \prime \prime}\left(u_{0}\right) \frac{u_{1}^{3}}{3 !}, \\
A_{4}= & N^{\prime}\left(u_{0}\right) u_{4}+N^{\prime \prime}\left(u_{0}\right)\left(\frac{u_{2}^{2}}{2 !}+u_{1} u_{3}\right) \\
& +N^{\prime \prime \prime}\left(u_{0}\right) \frac{u_{1}^{2} u_{2}}{2 !}+N^{(4)}\left(u_{0}\right) \frac{u_{1}^{4}}{4 !} .
\end{aligned}
$$

Several algorithms for the Adomian polynomials have been developed by Rach [30, 31], Adomian and Rach [32], Wazwaz [33], Biazar et al. [34], and several others. New, efficient algorithms with their subroutines written in Mathematica for rapid computer-generation of the Adomian polynomials have been provided by Duan in [35-37].

From (7)-(9) the solution components are determined by the classic Adomian recursion scheme:

$$
\begin{aligned}
u_{0}(x) & =\gamma(x), \\
u_{n+1}(x) & =-L^{-1} R u_{n}(x)-L^{-1} A_{n}(x), \quad n \geq 0 .
\end{aligned}
$$

Thus the $m$-term approximation $\phi_{m}(x)=\sum_{n=0}^{m-1} u_{n}(x)$ as obtained from the ADM can serve as the near-field approximation of the solution $u(x)$, where $x$ is in the neighborhood of the initial point $x=x_{0}$.

We remark that the convergence of the Adomian decomposition series has been previously proven by several researchers [30, 38-41]. For example, Abdelrazec and Pelinovsky [41] have recently published a rigorous proof of convergence for the ADM in accordance with the CauchyKovalevskaya theorem.

\section{Description of Adomian's Asymptotic Decomposition Method}

In this section, we advocate Adomian's asymptotic decomposition method for solving the Riccati equation. We remark 
that Adomian's asymptotic decomposition method does not need use of the initial condition to obtain the asymptotic solution or the solution in the large, which is another, convenient advantage in computations using this technique. Rather than nested integrations as required by decomposition, we now have nested differentiations. In effect our aim is to solve for the solution by not inverting the linear differential operator $L$, but instead by decomposing the nonlinear operator $\mathrm{Nu}$ and hence determining the asymptotic solution $u$. Toward this end, we rewrite (1) as

$$
r(x) u^{2}(x)=-p(x)-q(x) u(x)+u^{\prime}(x) .
$$

For the case when the coefficient $r(x) \neq 0$, we can divide both sides of (14) by $r(x)$, and we have

$$
u^{2}(x)=-\frac{p(x)}{r(x)}-\frac{q(x)}{r(x)} u(x)+\frac{1}{r(x)} u^{\prime}(x) .
$$

Substituting the respective decomposition series (i.e., $u(x)=$ $\sum_{n=0}^{\infty} u_{n}(x)$ and $\left.u^{2}(x)=\sum_{n=0}^{\infty} A_{n}(x)\right)$, we obtain

$$
\begin{aligned}
\sum_{n=0}^{\infty} A_{n}(x)= & -\frac{p(x)}{r(x)}-\frac{q(x)}{r(x)} \sum_{n=0}^{\infty} u_{n}(x) \\
& +\frac{1}{r(x)} \sum_{n=0}^{\infty} u_{n}^{\prime}(x),
\end{aligned}
$$

from which we design the asymptotic recursion scheme

$$
\begin{aligned}
A_{0}(x) & =-\frac{p(x)}{r(x)}, \\
A_{n+1}(x) & =-\frac{q(x)}{r(x)} u_{n}(x)+\frac{1}{r(x)} u_{n}^{\prime}(x), \quad n \geq 0 .
\end{aligned}
$$

We note that the Adomian polynomials $A_{n}$ for the quadratic nonlinearity $f(u)=u^{2}$ are

$$
\begin{gathered}
A_{0}=u_{0}^{2}, \\
A_{1}=2 u_{0} u_{1}, \\
A_{2}=2 u_{0} u_{2}+u_{1}^{2}, \\
A_{3}=2 u_{0} u_{3}+2 u_{1} u_{2}, \\
A_{4}=2 u_{0} u_{4}+2 u_{1} u_{3}+u_{2}^{2}, \\
\vdots \\
A_{n}=\sum_{i=0}^{n} u_{i} u_{n-i} .
\end{gathered}
$$

Using the form of the Adomian polynomials in (19), we rewrite the recursion schemes (17) and (18) as

$$
\begin{aligned}
u_{0}^{2}(x) & =-\frac{p(x)}{r(x)}, \\
\sum_{i=0}^{n+1} u_{i}(x) u_{n+1-i}(x) & =-\frac{q(x)}{r(x)} u_{n}(x)+\frac{1}{r(x)} u_{n}^{\prime}(x),
\end{aligned}
$$

In view of (21) and after appropriate manipulations, we obtain

$$
\begin{aligned}
\sum_{i=0}^{n+1} u_{i}(x) u_{n+1-i}(x)= & u_{0}(x) u_{n+1}(x) \\
& +\sum_{i=1}^{n} u_{i}(x) u_{n+1-i}(x) \\
& +u_{n+1}(x) u_{0}(x) \\
= & 2 u_{0}(x) u_{n+1}(x) \\
& +\sum_{i=1}^{n} u_{i}(x) u_{n+1-i}(x) .
\end{aligned}
$$

Consequently, with this result, the solution components are given by the following recursion scheme:

$$
\begin{aligned}
& u_{0}(x)=\sqrt{-\frac{p(x)}{r(x)}} \\
& u_{n+1}=\frac{1}{2 u_{0}(x)}\left(-\frac{q(x)}{r(x)} u_{n}(x)+\frac{1}{r(x)} u_{n}^{\prime}(x)\right. \\
& \left.-\sum_{i=1}^{n} u_{i}(x) u_{n+1-i}(x)\right), \quad n \geq 0 .
\end{aligned}
$$

Thus the obtained $m$-terms asymptotic approximation $\varphi_{m}(x)=\sum_{n=0}^{m-1} u_{n}(x)$ for the Riccati equation can serve as the far-field approximation, where $x$ is far from the initial point $x=x_{0}$.

\section{Numerical Examples}

In this section, several numerical examples are given to illustrate the efficiency of our technique as presented in this paper. We remark that all calculations are performed by Mathematica package 8 .

Example 1. Consider the following Riccati equation:

$$
u^{\prime}(x)+u^{2}(x)=1+x^{2}
$$

subject to initial condition $u(0)=1$.

The exact solution is known in advance to be

$$
u^{*}(x)=x+\frac{e^{-x^{2}}}{1+\int_{0}^{x} e^{-t^{2}} d t} .
$$

In Adomian's operator notation, we have

$$
L u+N u=g,
$$

where $L=d / d x, N=u^{2}$, and $g=1+x^{2}$.

To apply Adomian decomposition method, equation (26) should be written as the following,

$$
L u=g-N u \text {. }
$$


Applying the inverse operator $L^{-1}(\cdot)=\int_{0}^{x}(\cdot) d x$ to both sides yields

$$
u=u(0)+L^{-1} g-L^{-1} N u \text {. }
$$

Next we consider the solution as a series $u(x)=\sum_{n=0}^{\infty} u_{n}(x)$ and the nonlinearity $f(u)=u^{2}=\sum_{n=0}^{\infty} A_{n}(x)$, and upon substitution, we obtain

$$
\sum_{n=0}^{\infty} u_{n}(x)=u(0)+L^{-1} g-L^{-1} \sum_{n=0}^{\infty} A_{n}(x) .
$$

The components of the series solution are given by the recursion scheme

$$
\begin{aligned}
u_{0} & =u(0)+L^{-1} g, \\
u_{n+1} & =-L^{-1} A_{n}(x), \quad n \geq 0 .
\end{aligned}
$$

The first few components are as follows:

$$
\begin{aligned}
u_{0} & =1+x+\frac{x^{3}}{x} \\
u_{1} & =-x-x^{2}-\frac{x^{3}}{3}-\frac{x^{4}}{6}-\frac{2 x^{5}}{15}-\frac{x^{7}}{63} \\
u_{3} & =x^{2}+\frac{4 x^{3}}{3}+\frac{2 x^{4}}{3}+\frac{x^{5}}{3}+\frac{19 x^{6}}{90}+\frac{22 x^{7}}{315}+\frac{x^{8}}{56} \\
& +\frac{38 x^{9}}{2835}+\frac{2 x^{11}}{2079},
\end{aligned}
$$

The partial sums $\phi_{m}(x)=\sum_{n=0}^{m-1} u_{n}(x)$ of the Adomian decomposition series can serve as a near-field approximate solution.

Solving (26) by Adomian's asymptotic decomposition method, we first rewrite it as

$$
N u=g-L u \text {. }
$$

Next we assume the series $u=\sum_{n=0}^{\infty} u_{n}$ and the nonlinearity $u^{2}=\sum_{n=0}^{\infty} A_{n}(x)$.

Upon substitution and using the form of the Adomian polynomials in (19), we obtain the solution components of the far-field approximation $\varphi_{n}(x)$ according to the recursion scheme (23)

$$
\begin{gathered}
u_{0}=\sqrt{1+x^{2}}, \\
u_{1}=-\frac{x}{2\left(1+x^{2}\right)}, \\
u_{2}=\frac{2-3 x^{2}}{8\left(1+x^{2}\right)^{5 / 2}},
\end{gathered}
$$

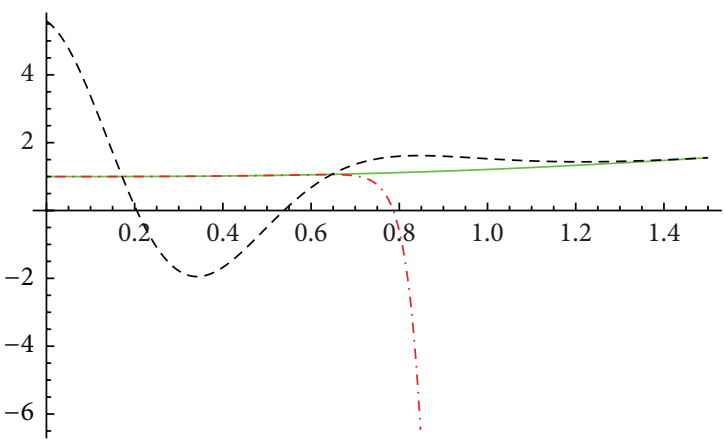

FIGURE 1: The near-field approximation $\phi_{20}(x)$ (dot-dashed line), far-field approximation $\varphi_{7}(x)$ (dashed line), and exact solution $u^{*}(x)$ (solid line)

from which we conclude that $u(x) \sim x$ is the slant asymptote of the exact solution; that is, $\lim _{x \rightarrow \infty}\left[u^{*}(x)-x\right]=0$.

Computation shows that this Adomian decomposition series has a finite radius of convergence. By plotting the curves of $\phi_{m}(x)$ and $\varphi_{n}(x)$ for several values of $m$ and $n$, we do not find any regions of overlap. In this case, we connect the two approximations by the Padé approximant of $\phi_{m}(x)$ or simply replace $\phi_{m}(x)$ by its Padé approximant and then match the Padé approximant with $\varphi_{n}(x)$. For example, we investigate $\phi_{20}$ and $\varphi_{7}$. The curves of the near-field approximation $\phi_{20}(x)$, the far-field approximation $\varphi_{7}(x)$, and the exact solution $u^{*}(x)$ are plotted in Figure 1.

We calculated the Padé approximant $[9 / 10]\left\{\phi_{20}(x)\right\}$ by Mathematica and found that the Padé approximant $[9 / 10]\left\{\phi_{20}(x)\right\}$ and the far-field approximation $\varphi_{7}(x)$ overlap in the approximate region $1.5 \leq x \leq 5.5$; see Figure 2. Thus we can match them as

$$
\tilde{u}(x)=\left[\frac{9}{10}\right]\left\{\phi_{20}(x)\right\} h(\xi-x)+\varphi_{7}(x) h(x-\xi),
$$

which is a global approximation, where $\xi$ belongs to the region of overlap and $h(x)$ is the unit step function; that is,

$$
h(x)= \begin{cases}1, & x<0 \\ \frac{1}{2}, & x=0 \\ 1, & x>0\end{cases}
$$

Example 2. Consider the following Riccati equation:

$$
u^{\prime}(x)+u^{2}(x)=1
$$

with the initial value $u(0)=0$.

The exact solution is known in advance to be

$$
u^{*}(x)=\tanh x
$$

By the Adomian decomposition method and applying the integral operator $L^{-1}(\cdot)=\int_{0}^{x}(\cdot) d x$, we have

$$
u=x-L^{-1} u^{2} .
$$




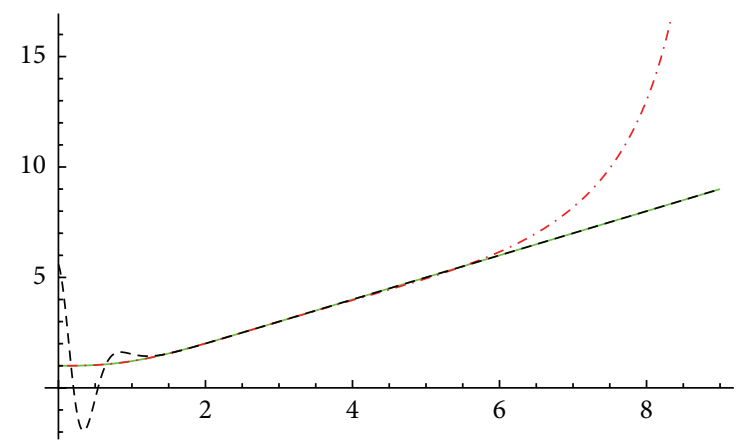

Figure 2: The Padé approximant $[9 / 10]\left\{\phi_{20}(x)\right\}$ (dot-dashed line), far-field approximation $\varphi_{7}(x)$ (dashed line), and exact solution $u^{*}(x)$ (solid line).

As before, we decompose $u$ and $u^{2}$ as

$$
\begin{gathered}
u=\sum_{n=0}^{\infty} u_{n}, \\
u^{2}=\sum_{n=0}^{\infty} A_{n} .
\end{gathered}
$$

Thus the solution components of the near-field approximation $\phi_{m}(x)$ are determined recursively as

$$
\begin{gathered}
u_{0}=x, \\
u_{1}=-\frac{x^{3}}{3}, \\
\vdots \\
u_{n}=-\int_{0}^{x} A_{n-1} d x, \quad n \geq 1 .
\end{gathered}
$$

By Adomian's asymptotic decomposition method according to the recursion scheme (23), the solution components of the far-field approximation $\varphi_{n}(x)$ are computed as

$$
\begin{aligned}
& u_{0}=1, \\
& u_{1}=0, \\
& u_{2}=0,
\end{aligned}
$$

from which we conclude that $u(x)=1$ is the horizontal asymptote of the exact solution when the independent variable $x$ approaches infinity.

The curves of the near-field approximation $\phi_{20}(x)$, the farfield approximation $\varphi_{3}(x)$, and the exact solution $u^{*}(x)$ are plotted in Figure 3.

We calculated the Padé approximant [13/12] $\left\{\phi_{20}(x)\right\}$ by Mathematica and found that the Padé approximant

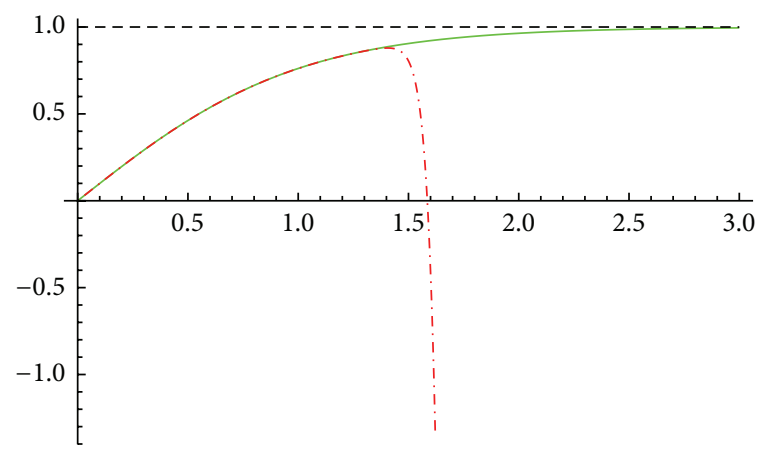

FIGURE 3: The near-field approximation $\phi_{20}(x)$ (dot-dashed line), far-field approximation $\varphi_{3}(x)$ (dashed line), and exact solution $u^{*}(x)$ (solid line).

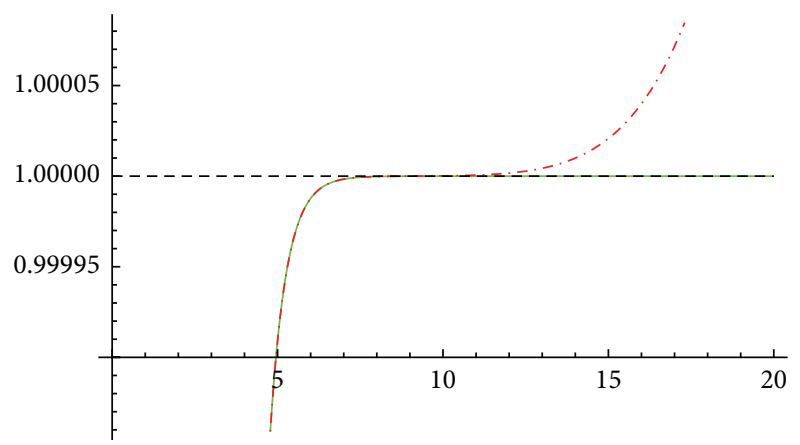

FIgURe 4: The Padé approximant [13/12] $\left\{\phi_{20}(x)\right\}$ (dot-dashed line), far-field approximation $\varphi_{3}(x)$ (dashed line), and exact solution $u^{*}(x)$ (solid line).

$[13 / 12]\left\{\phi_{20}(x)\right\}$ and the far-field approximation $\varphi_{3}(x)$ overlap almost in the approximate region $7<x<11$; see Figure 4. Thus we can match them as

$$
\widetilde{u}(x)=\left[\frac{13}{12}\right]\left\{\phi_{20}(x)\right\} h(\xi-x)+\varphi_{3}(x) h(x-\xi),
$$

which is a global approximation, where $\xi$ belongs to the region of overlap.

\section{Conclusion}

In this work, we combined the ADM and the AADM to approximate the global solution of the Riccati equation. We evaluated the approximate solution by matching the Padé approximant of the near-field approximation derived from the ADM with the far-field approximation derived from the AADM. Furthermore we have shown that the AADM can be an important complement in analysis of the solution's asymptote.

\section{Conflict of Interests}

The authors declare that there is no conflict of interests regarding the publication of this paper. 


\section{References}

[1] W. T. Reid, Riccati Differential Equations, Academic Press, New York, NY, USA, 1972.

[2] E. S. Fraga, The Schrödinger and Riccati Equations, vol. 70 of Lecture Notes in Chemistry, Springer, Berlin, Germany, 1999.

[3] R. Shankar, Principles of Quantum Mechanics, Plenum, New York, NY, USA, 1980.

[4] A. Khare and U. Sukhatme, Supersymmetry in Quantum Mechanics, World Scientific, Singapore, 2001.

[5] M. I. Zelekin, Homogeneous Spaces and Riccati Equation in Variational Calculus, Factorial, Moscow, Russia, 1998, (Russian).

[6] V. B. Matveev and M. A. Salle, Darboux Transformations and Solitons, Springer Series in Nonlinear Dynamics, Springer, Berlin, Germany, 1991.

[7] I. L. Buchbinder, S. D. Odintsov, and I. L. Shapiro, Effective Action in Quantum Gravity, IOP Publishing, 1992.

[8] K. Milton, S. D. Odintsov, and S. Zerbini, "Bulk versus brane running couplings," Physical Review D, vol. 65, Article ID 065012, 2002.

[9] H. C. Rosu and F. A. de la Cruz, "One-parameter Darbouxtransformed quantum actions in thermodynamics," Physica Scripta, In press.

[10] J. F. Cariñena, G. Marmo, A. M. Perelomov, and M. F. Rañada, "Related operators and exact solutions of Schrödinger equations," International Journal of Modern Physics A, vol. 13, no. 28, pp. 4913-4929, 1998.

[11] B. D. Anderson and J. B. Moore, Optimal Control-Linear Quadratic Methods, Prentice-Hall, Upper Saddle River, NJ, USA, 1999.

[12] I. Lasiecka and R. Triggiani, Differential and Algebraic Riccati Equations with Application to Boundary/Point Control Problems: Continuous Theory and Approximation Theory, vol. 164 of Lecture Notes in Control and Information Sciences, Springer, Berlin, Germany, 1991.

[13] G. Adomian, Nonlinear Stochastic Operator Equations, Academic Press, Orlando, Fla, USA, 1986.

[14] G. Adomian and R. Rach, "On linear and nonlinear integrodifferential equations," Journal of Mathematical Analysis and Applications, vol. 113, no. 1, pp. 199-201, 1986.

[15] G. Adomian, Solving Frontier Problems of Physics: The Decomposition Method, Kluwer Academic, Dordrecht, The Netherlands, 1994.

[16] G. Adomian and R. Rach, "On the solution of algebraic equations by the decomposition method," Journal of Mathematical Analysis and Applications, vol. 105, no. 1, pp. 141-166, 1985.

[17] A. M. Wazwaz, Partial Differential Equations and Solitary Waves Theory, Higher Education Press, Beijing, China, Springer, Berlin, Germany, 2009.

[18] A.-M. Wazwaz, Linear and Nonlinear Integral Equations: Methods and Applications, Higher Education Press, Beijing, China, 2011.

[19] H. Bulut and D. J. Evans, "On the solution of the Riccati equation by the decomposition method," International Journal of Computer Mathematics, vol. 79, no. 1, pp. 103-109, 2002.

[20] M. A. El-Tawil, A. A. Bahnasawi, and A. Abdel-Naby, "Solving Riccati differential equation using Adomian's decomposition method," Applied Mathematics and Computation, vol. 157, no. 2, pp. 503-514, 2004.
[21] P.-Y. Tsai and C.-K. Chen, "An approximate analytic solution of the nonlinear Riccati differential equation," Journal of the Franklin Institute, vol. 347, no. 10, pp. 1850-1862, 2010.

[22] A. R. Vahidi and M. Didgar, "An improved method for determining the solution of Riccati equations," Neural Computing and Applications, vol. 23, no. 5, pp. 1229-1237, 2013.

[23] A. R. Vahidi, Z. Azimzadeh, and M. Didgar, "An efficient method for solving Riccati equation using homotopy perturbation method," Indian Journal of Physics, vol. 87, no. 5, pp. 447454, 2013.

[24] A. R. Vahidi, M. Didgar, and R. C. Rach, "An improved approximate analytic solution for Riccati equations over extended intervals," Indian Journal of Pure and Applied Mathematics, vol. 45, no. 1, pp. 27-38, 2014.

[25] G. Adomian, "An investigation of the asymptotic decomposition method for nonlinear equations in physics," Applied Mathematics and Computation, vol. 24, no. 1, pp. 1-17, 1987.

[26] G. Adomian, "An adaptation of the decomposition method for asymptotic solutions," Mathematics and Computers in Simulation, vol. 30, no. 4, pp. 325-329, 1988.

[27] G. Adomian, "Solving the nonlinear equations of physics," Computers \& Mathematics with Applications, vol. 16, no. 10-11, pp. 903-914, 1988.

[28] R. Rach and J.-S. Duan, "Near-field and far-field approximations by the Adomian and asymptotic decomposition methods," Applied Mathematics and Computation, vol. 217, no. 12, pp. 59105922,2011

[29] K. Haldar and B. K. Datta, "Integrations by asymptotic decomposition," Applied Mathematics Letters, vol. 9, no. 2, pp. 81-83, 1996.

[30] R. C. Rach, "A new definition of the Adomian polynomials," Kybernetes, vol. 37, no. 7, pp. 910-955, 2008.

[31] R. Rach, "A convenient computational form for the Adomian polynomials," Journal of Mathematical Analysis and Applications, vol. 102, no. 2, pp. 415-419, 1984.

[32] G. Adomian and R. Rach, "Generalization of Adomian polynomials to functions of several variables," Computers \& Mathematics with Applications, vol. 24, no. 5-6, pp. 11-24, 1992.

[33] A.-M. Wazwaz, "A new algorithm for calculating Adomian polynomials for nonlinear operators," Applied Mathematics and Computation, vol. 111, no. 1, pp. 53-69, 2000.

[34] J. Biazar, E. Babolian, G. Kember, A. Nouri, and R. Islam, "An alternate algorithm for computing Adomian polynomials in special cases," Applied Mathematics and Computation, vol. 138, no. 2-3, pp. 523-529, 2003.

[35] J.-S. Duan, "Recurrence triangle for Adomian polynomials," Applied Mathematics and Computation, vol. 216, no. 4, pp. 12351241, 2010.

[36] J.-S. Duan, "An efficient algorithm for the multivariable Adomian polynomials," Applied Mathematics and Computation, vol. 217, no. 6, pp. 2456-2467, 2010.

[37] J.-S. Duan, "Convenient analytic recurrence algorithms for the Adomian polynomials," Applied Mathematics and Computation, vol. 217, no. 13, pp. 6337-6348, 2011.

[38] Y. Cherruault and G. Adomian, "Decomposition methods: a new proof of convergence," Mathematical and Computer Modelling, vol. 18, no. 12, pp. 103-106, 1993.

[39] K. Abbaoui and Y. Cherruault, "Convergence of Adomian's method applied to differential equations," Computers \& Mathematics with Applications, vol. 28, no. 5, pp. 103-109, 1994. 
[40] K. Abbaoui and Y. Cherruault, "New ideas for proving convergence of decomposition methods," Computers \& Mathematics with Applications, vol. 29, no. 7, pp. 103-108, 1995.

[41] A. Abdelrazec and D. Pelinovsky, "Convergence of the Adomian decomposition method for initial-value problems," Numerical Methods for Partial Differential Equations, vol. 27, no. 4, pp. 749766, 2011. 


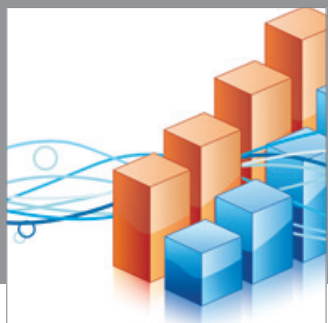

Advances in

Operations Research

mansans

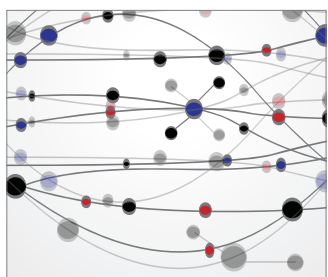

The Scientific World Journal
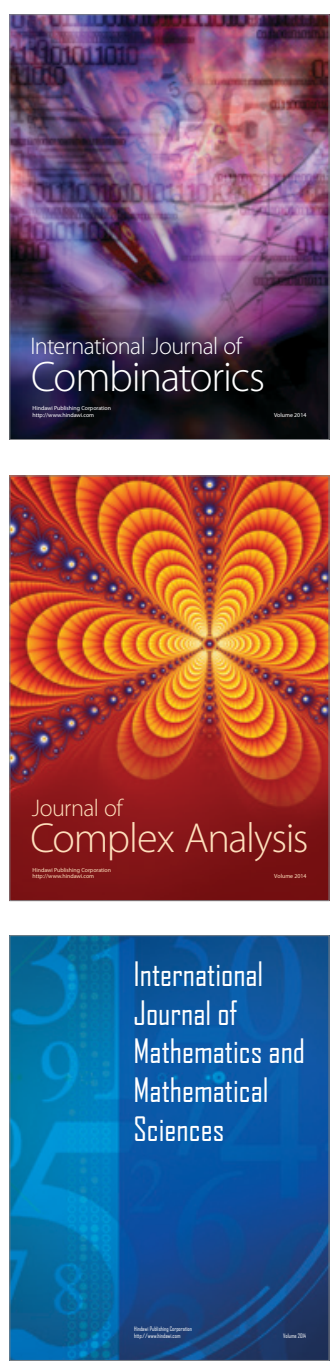
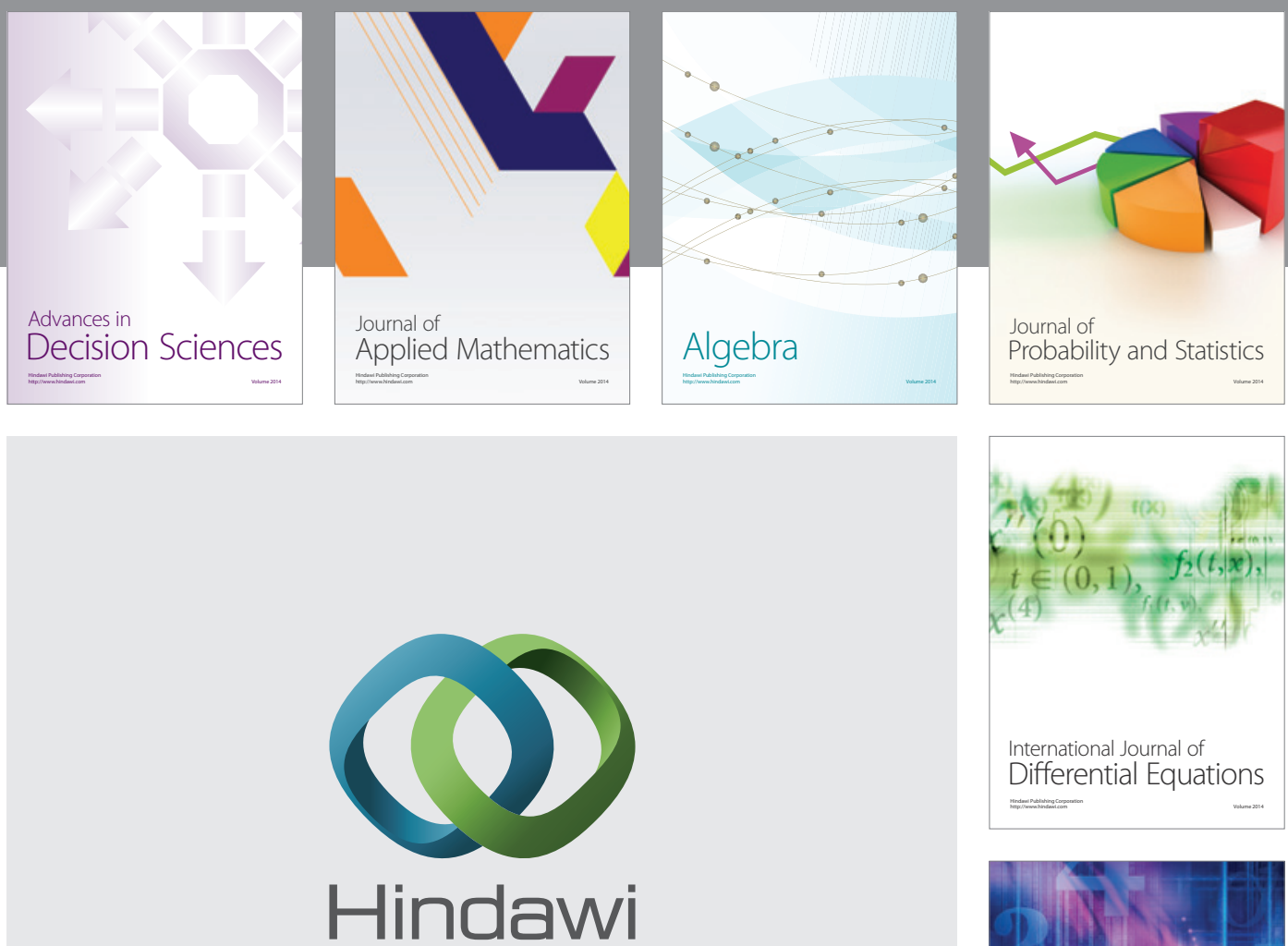

Submit your manuscripts at http://www.hindawi.com
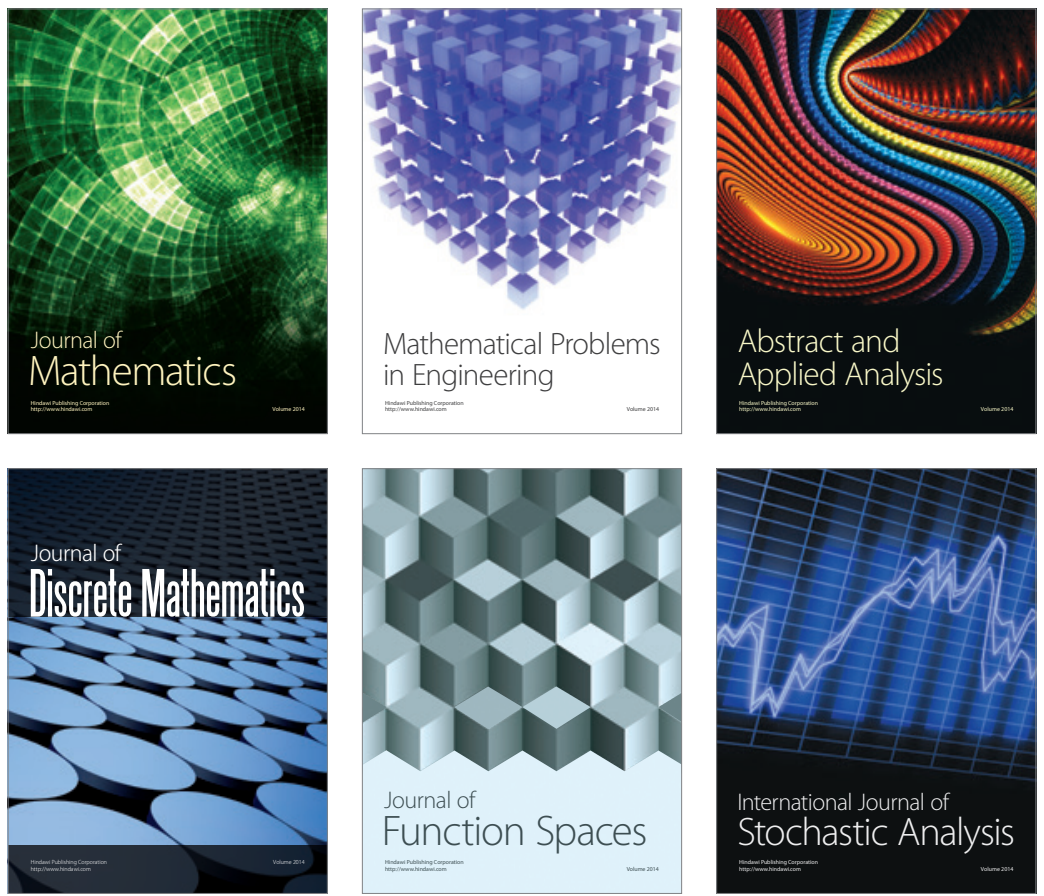

Journal of

Function Spaces

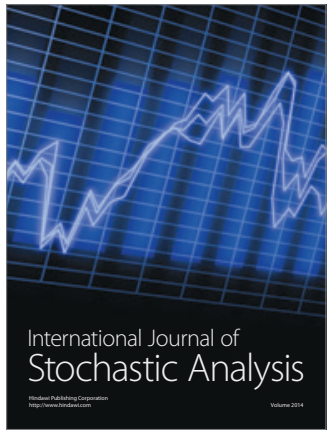

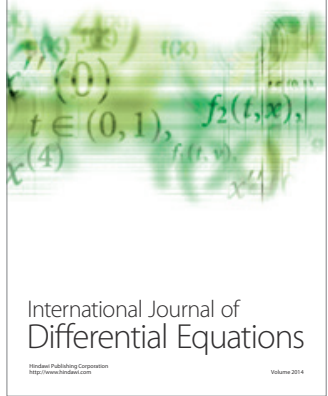
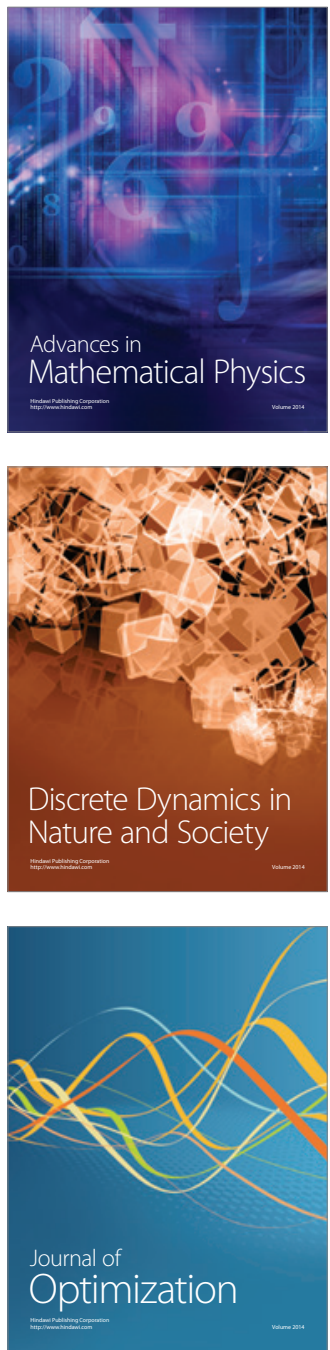